\title{
Natural treatment for anxiety
}

\section{Editorial}

If you suffer from extreme stress or anxiety, use natural deep breathing, biofeedback, full hydration and taking Omega 3 fatty acid with $1000 \mathrm{DHA}+\mathrm{EPA}$, minerals \& vitamins with high bioavailability and B complex high potency supplements. For some individuals, medications can be temporary treatment but with severe side effects. Weigh the risks vs benefits and remember that Meditation is better than medications. Commonly prescribed drugs include antidepressants, such as selective serotonin reuptake inhibitors (like Prozac or Zoloft), or dual serotonin and norepinephrine reuptake inhibitors (like Effexor or Cymbalta). These drugs take longer to work than the traditional anti-anxiety drugs but also may provide greater symptom relief over time. Withdrawal symptoms and possible addiction should be noted. Follow the wellness IQ section and modify your diet which is the best natural treatment for anxiety. Being depressed can make you feel helpless. You're not. Along with therapy and sometimes medication, there's a lot you can do on your own to fight back. Changing your behavior - your physical activity, lifestyle, and even your way of thinking - are all natural depression treatments.

Do you want to learn natural depression treatments that can help you feel better starting right now? Here are some tips.

i. If you are depressed, you need a routine, Depression can strip away the structure from your life. One day melts into the next. Setting a gentle daily schedule can help you get back on track

ii. Set goals: When you are depressed, you may feel like you cannot accomplish anything. That makes you feel worse about yourself. To push back, set daily goals for yourself. "Start very small. "Make your goal something that you can succeed at, like doing the dishes every other day." As you start to feel better, you can add more challenging daily goals.

iii. Exercise: Exercise temporarily boosts feel-good chemicals called endorphins. It may also have long-term benefits for people with depression. Regular physical activity seems to encourage the brain to rewire itself in positive ways, Cook says. How much exercise do you need? You do not need to run marathons to get a benefit. Just walking a few times a week can help.

iv. Eat healthy: There is no magic diet that fixes depression, but watching what you eat is a good idea. If depression tends to make you overeat, getting in control of your eating will help you feel better. Although nothing is definitive, Cook says there's evidence that foods with omega-3 fatty acids such as salmon and tuna - and folic acid - such as spinach and avocado - could help ease depression.

v. Get enough sleep: Depression can make it hard to get enough sleep, and not getting enough sleep can make depression worse. What can you do? Start by making some changes to your lifestyle. Go to bed and get up at the same time every day. Try not to nap. Take all the distractions out of your bedroom - no computer and no TV. In time, you may find your sleep improves.

vi. Take on responsibilities: When you are depressed, you may want to pull back from life and give up your responsibilities at home and at work. Do not. Staying involved and having daily responsibilities
Volume 2 Issue 3 - 2015

George Grant
World organization of Natural Medicine, Canada

Correspondence: George Grant, World organization of Natural Medicine, Richmond Hill, Ontario, Canada,Tel 4I6 562 3140, Email drgrant@rogers.com

Received:June II, 20I5 | Published: June 15, 2015

can work as a natural depression treatment. They ground you and give you a sense of accomplishment. If you are not up to full-time school or work, that is fine. Think about part-time. If that seems like too much, consider volunteer work.

vii. Challenge negative thoughts: In your fight against depression, a lot of the work is mental - changing how you think. When you are depressed, you leap to the worst possible conclusions. The next time you are feeling terrible about yourself, use logic as a natural depression treatment. You might feel like no one likes you, but is there real evidence for that? You might feel like the most worthless person on the planet, but is that really likely? It takes practice, but in time you can beat back those negative thoughts before they get out of control.

viii. Check with your doctor before using supplements: "There are promising evidence for certain supplements for depression," says Cook, such as fish oil, folic acid, and SAMe. However, more research needs to be done before we will know for sure. Always check with your doctor before starting any supplement, especially if you are already taking medications.

ix. Do something new: When you are depressed, you are in a rut. Push yourself to do something different. Go to a museum. Pick up a used book and read it on a park bench. Volunteer at a soup kitchen. Take a language class. When we challenge ourselves to do something different, there are chemical changes in the brain. "Trying something new alters the levels of dopamine, which is associated with pleasure, enjoyment, and learning."

x. Antidote for anxiety: I feel protection, connection, love and a sense of myself as having the capacity to call in something beautiful and holy in the moment.

\section{Acknowledgements}

None.

\section{Conflict of interest}

The author declares no conflict of interest. 